\title{
Enquête sur les formations initiales et continues en EHST dans les IUFM
}

\section{Pierre Savaton}

\section{(2) OpenEdition \\ Journals}

Édition électronique

URL : http://journals.openedition.org/trema/66

DOI : 10.4000/trema.66

ISSN : 2107-0997

Éditeur

Faculté d'Éducation de l'université de Montpellier

Édition imprimée

Date de publication : 1 octobre 2006

Pagination : 11-19

ISSN : 1167-315X

Référence électronique

Pierre Savaton, «Enquête sur les formations initiales et continues en EHST dans les IUFM », Tréma [En ligne], 26 | 2006, mis en ligne le 11 janvier 2010, consulté le 20 avril 2019. URL : http:// journals.openedition.org/trema/66; DOI : 10.4000/trema.66

Ce document a été généré automatiquement le 20 avril 2019

Trema 


\title{
Enquête sur les formations initiales et continues en EHST dans les IUFM
}

\author{
Pierre Savaton
}

1 En vue des Journées de Montpellier, le comité de programme avait décidé de lancer une enquête auprès des IUFM sur les formations initiales et continues en Histoire des sciences qu'ils organisent en leur sein. Cette enquête cherchait à recueillir des éléments de description pour un état des lieux de la formation en HST des enseignants. Elle a pris la forme d'un questionnaire fort simple, envoyé aux IUFM par voie électronique avec une courte présentation du groupe auteur de cette enquête et de ses finalités. Les Journées de Montpellier ayant reçu l'appui de la Commission des Directeurs d'IUFM (CDIUFM), cette enquête a été diffusée officiellement par la Communication de la CDIUFM aux chargés de communication des différents instituts. Cette diffusion a parfois été doublée par des envois individuels à des formateurs IUFM avec lesquels nous avions des contacts privés ou professionnels.

2 La construction du questionnaire (questions en annexe1) était déterminée par quelques questions simples touchant aux effectifs concernés par ces formations, au nombre d'heures assurées, aux effectifs de formateurs impliqués, aux modalités pédagogiques mises en oeuvre, à la place dans les plans de formation et aux rapports avec d'autres formations. Ce questionnaire envoyé sans accompagnement laissait volontairement libres de leur définition d'une formation en HST, ceux qui y ont répondu. Il nous avait semblé en effet préférable pour un premier contact de laisser libre de sa définition celui qui répond pour prendre note de ce qui se fait quels qu'en soient les modalités, objectifs et contenus, postulant, et semble-t-il avec raison, que la diversité des initiatives souvent individuelles et spontanées ne pouvait qu'aboutir à une grande diversité de contenus et de définitions. En effet, ce questionnaire visant à fournir à la fois des éléments de description des pratiques affichées et à établir des liens avec des formateurs auteurs d'initiatives locales non inventoriées, aurait risqué de manquer sa cible en rejetant par avance des expériences originales.

3 Sur les 31 IUFM, 20 ont transmis une réponse, qu'on trouvera en annexe 2. Ces réponses sont parfois partielles car limitées à un site de l'IUFM, un type de formation (formation 
des professeurs d'écoles par exemple) ou une discipline (mathématiques par exemple). Elles nous ont été transmises par le chargé de communication de l'IUFM ou par un formateur sans qu'il nous soit possible de déterminer l'exhaustivité de l'inventaire établi. Cette enquête permet toutefois un premier aperçu d'une situation jusqu'alors non décrite et nous indique quelques grandes caractéristiques de la formation en HST dans les IUFM. Ces retours nous ont permis également d'établir un premier contact avec des formateurs et d'envisager la mise en place d'un réseau d'échange ou d'une liste de diffusion d'informations sur la formation en EHST.

\section{Les résultats de l'enquête}

Sur les 20 IUFM qui nous ont répondu, 18 déclarent assurer au moins un stage ou une formation portant sur l'HST ou s'appuyant d'une manière ou d'une autre sur des apports de l'HST. Ce chiffre est bien sûr à prendre avec les réserves soulevées précédemment, mais est un premier indicateur qui pourrait être revu à la hausse compte tenu de l'absence de réponses de quelques IUFM susceptibles d'assurer au moins une formation de ce type, compte tenu de leur taille, de la présence d'historiens des sciences au sein de l'établissement ou de l'existence d'un laboratoire d'histoire des sciences dans l'université voisine. Les conditions de mise en oeuvre et de réalisation de la formation continue étant variables d'une académie à une autre et liées à des choix nationaux et rectoraux indépendants des projets d'établissement et plan de formation des IUFM, nous présenterons distinctement l'état des lieux des formations initiale et continue, tel qu'il nous est possible de le tracer à partir de ce questionnaire. Les résultats sont présentés sans grands commentaires pour éviter toute interprétation excessive et fournir des éléments de réflexion aux participants de ces Journées.

\section{L'Histoire des sciences et des techniques dans la formation initiale.}

6 La plupart des IUFM déclarent proposer des formations s'appuyant à divers degrés sur l'HST. Ces réponses positives couvrent une diversité de situations allant d'une unique formation de l'ordre de 3 à 6 heures à une offre spécifique de formation en PE1, PE2, PLC1, PLC2, PLP2.2. ${ }^{1}$

Des éléments d'histoire des sciences figurent ainsi dans la formation des PE1, soit au sein de la préparation à l'épreuve de sciences (Bourgogne, Franche-Comté, Créteil), soit au sein de la formation générale dans le cadre par exemple d'un module d'épistémologie des savoirs scolaires (Lyon/St Etienne). C'est un enseignement de quelques heures qui ne concerne que peu d'étudiants lorsqu'il existe. Il n'y a donc pas véritablement d'enseignement d'HST en PE1. La situation est semblable en PE2. Signalons 6 heures consacrées à l'épistémologie et l'histoire des sciences dans le cadre d'une formation à la méthodologie du mémoire professionnel (Créteil) et 15 heures d'HST dans le cadre de la 
formation commune avec les PLC2 et PLP2.2 (Basse-Normandie). L'HST ne fait donc pas partie de la formation initiale des professeurs d'écoles.

8 Le bilan est du même ordre en PLC1 où la formation se réduit à la préparation des épreuves des concours, or ceux-ci ne font aucune place explicite à l'histoire des sciences. Signalons l'introduction à Créteil de 3 heures sur l'histoire des mathématiques dans le cadre de la préparation de l'épreuve sur dossier, 3 à 6 heures en SVT et SPC ${ }^{2}$ en Poitou Charente, et 20 heures d'histoire des sciences du vivant en lien avec la didactique des SVT à Rouen.

9 L'HST, lorsqu'elle figure dans les plans de formation initiale, est généralement proposée dans les formations des PLC2 Math, SPC, SVT et PLP2.2. Math/sciences : 19 formations sont déclarées par les IUFM. Les disciplines n'y ont pas la même place : 9 SPC, 7 Maths, 3 SVT et 7 PLP2.2 Maths-sciences. Ces formations durent de 6 heures (Créteil, Toulouse, Orléans-Tours, Bretagne, Versailles) à 9h (Aix-Marseille, Bourgogne), voire $12 \mathrm{~h}$ (Guadeloupe, Créteil) ou 15h (Créteil, Basse-Normandie).

Les formations sont assurées par 1 ou 2 formateurs; dans quelques cas, elles font appel à plusieurs conférenciers et formateurs. En règle générale (11/14) les formations en HST s'inscrivent dans le plan de formation disciplinaire ; en Basse-Normandie elles le sont également dans le plan de la formation commune ${ }^{3}$.

11 Les stages et formations en HST s'appuient (9/9) sur des études de textes et de documents ou des exposés ou conférences avec souvent (4/9) des ateliers de production de séquences d'enseignement. Deux formations correspondent à une série de conférences. Ces formations sont majoritairement déclarées (10 IUFM) comme au service de la formation disciplinaire en sciences, en liaison avec les aspects didactiques et épistémologiques de la discipline plus qu'avec ses aspects historiques. Quelques formations sont proposées comme un enseignement de l'histoire des sciences et des techniques comme une discipline à part entière ou comme relevant d'une culture scientifique.

Il convient de noter qu'un nombre important des formateurs qui ont répondu à ce questionnaire (une majorité ?) assurent des formations en didactique des sciences et sont eux même engagés dans des recherches en didactique des sciences expérimentales ou membre d'un IREM ${ }^{4}$. Ce rapprochement pressenti entre l'histoire des sciences et des techniques et la didactique des sciences déterminait l'orientation de l'Atelier 3 de ces Journées de Montpellier.

\section{L'Histoire des sciences et des techniques en formation continue}

Les plans de formation continue étant parfois très variables d'une année à la suivante, les réponses collectées décrivent indifféremment des stages pérennes et des stages une seule fois réalisés. Certains figurent aux plans 2004-2005, d'autres en ont été retirés. La constitution des plans de formation continue, départementaux ou académiques, relevant de logiques et de démarches parfois opposées (logique de l'offre/demande de formation, démarche centralisée ou collective) il est difficile de tirer de ces réponses plus d'informations que la seule existence ou non d'un stage. 
Dix IUFM indiquent la présence dans le plan de formation de leur académie de formations en HST, mais plusieurs déplorent dans leur réponse la disparition de ces stages depuis un an ou deux.

Sans préjuger une fois de plus de leur contenu, nous pouvons signaler 3 cas de FC en HST à destination des enseignants du primaire. C'est le cas à Besançon avec un stage de 78 heures qui a concerné 13 stagiaires et 10 formateurs. Il articulait des études de textes, des exposés, des visites et des recherches personnelles. Sur Versailles, 6 heures d'histoire de l'enseignement des mathématiques ont également été proposées et une expérience semblable a eu lieu en 2001-2002 en Poitou - Charente. Il est manifeste que l'HST ne fait pas partie de la formation continue des enseignants du Primaire.

situation est différente pour le Secondaire où 29 stages sont déclarés (Aix-Marseille, Bourgogne, Bretagne, Basse-Normandie, Créteil, Limousin, Lyon, Orléans-Tours, Poitou Charente) avec une majorité de stages portant sur l'histoire des mathématiques. Cette situation est à mettre en relation avec le travail des IREM. Ces formations de 6 à 18 heures touchent de 30 personnes à plus d'une centaine et font appel à 2 formateurs, voire jusqu'à 10 intervenants selon les dispositifs et les IUFM. Elles s'inscrivent dans les plans de formation au sein des dispositifs disciplinaires et transversaux. Dans 2 IUFM, l'HST figure comme enseignement d'une discipline à part entière (Bretagne, Poitou - Charente) ; dans 5 IUFM, elle relève d'une recherche de culture personnelle scientifique (Poitou Charente, Basse-Normandie, Aix-Marseille, Bretagne, Bourgogne). Elle est enfin pensée, comme en formation initiale, au service de l'enseignement disciplinaire, en liaison avec les aspects didactiques, épistémologiques et historiques de la discipline.

7 Quelques séminaires en HST, internes aux IUFM et destinés aux formateurs, sont également signalés par les IUFM de Bretagne, Basse-Normandie et Bourgogne. Créteil propose également un stage en HST validable comme module de DEA de didactique (master 2 recherche) par l'université Paris 7.

Les modalités de ces stages sont les mêmes la plupart du temps que celles des formations PLC2, PLP2.2, à savoir : études de textes et de documents, exposés, conférences et ateliers de production de séquences d'enseignement. Quelques-uns y ajoutent des recherches individuelles et des visites de musées. 
19 La diversité des situations est particulièrement marquée en ce qui concerne la FC. Certains IUFM déclarent un choix parfois conséquent de formations en HST, là où d'autres ne signalent aucune formation. Les conditions de mise en œuvre et de réalisation de formations en HST dans le cadre de la formation continue méritent également que l'on s'y attarde au cours de ces journées. Il est étonnant par exemple de constater que certaines académies qui pourraient s'appuyer sur l'existence locale d'un laboratoire en histoire des sciences et des techniques ne proposent aucune formation aux enseignants $\mathrm{du}$ primaire et du secondaire. L'atelier 1 reviendra probablement sur cet aspect.

\section{Bilan et perspective}

La lecture des résultats de cette enquête confirme la situation déjà ressentie en mai 2004 au Colloque de la Société Française d'Histoire des Sciences et des Techniques (SFHST) à Poitiers, à savoir : l'histoire des sciences et des techniques n'est guère enseignée aux enseignants, que ce soit en formation initiale à l'université ou dans les IUFM, ou en formation continue. En même temps elle témoigne de l'existence de formations et de formateurs en HST dans la plupart des IUFM, ce qui montre l'existence de potentialités et peut faire augurer d'une reconnaissance du rôle de l'HST dans la formation des enseignants.

21 La nature et l'organisation de ces formations montrent l'importance et l'impact de l'action des IREM. Elle montre enfin un lien majeur avec l'enseignement et la didactique des disciplines.

Cette enquête doit être poursuivie de manière plus structurée, non seulement pour préciser ces premières impressions, mais également pour l'effet que la connaissance des pratiques des uns et des autres peut avoir sur la pratique de tous. Le groupe qui a organisé ces Journées de Montpellier défend effectivement l'idée de développer les échanges entre formateurs en HST dans les IUFM pour développer la mise en œuvre et la réalisation de formations. La constitution d'un réseau de formateurs nous semble être un élément d'appui pour défendre le rôle et la place de l'HST dans la formation des enseignants. D'ores et déjà une liste de diffusion est ouverte sur le site de l'IUFM de Poitiers et une nouvelle enquête se construit.

Ces Journées devraient nous permettre de préciser également cet aperçu de la place de l'HST dans les formations des IUFM. 


\section{ANNEXES}

\section{Annexe 1}

Questions du questionnaire envoyé aux IUFM début 2005

\section{HST en formation initiale}

Y-a-t-il des formations en HST ou empruntant à l'HST dans votre IUFM?

Identification du public concerné :

- PE1,

- PE2,

- PLC1 (précisez les disciplines) :

- PLC2 (précisez les disciplines) :

- PLP2.2. (précisez les disciplines) :

- Formateurs

Nombre d'heures de formation:

Nombre de personnes en formation :

Nombre de formateurs intervenant dans ces formations :

Place dans les plans de formations

Les formations en HST sont assurées sur les heures :

- Des formations disciplinaires des plans de formation?

- Des formations transversales, communes?

- De la formation générale?

Modalités des stages et formations en HST

- Etude de textes et documents

- Présentation, exposé, conférence

- Atelier de production de séquences d'enseignement

- Autre (précisez) :

Articulation formation en HST et formation disciplinaire

- Formation en HST comme discipline à part entière

- Formation en HST au service de la formation disciplinaire scientifique

- en liaison avec les aspects didactiques de la discipline

- en liaison avec les aspects épistémologiques de la discipline

- en liaison avec les aspects historiques de la discipline 


\section{HST en formation continue}

Y-a-t-il des formations en HST ou empruntant à l'HST assurées par l'IUFM dans le cadre de la formation continue?

Identification du public :

- Instituteurs et PE,

- Enseignants des collèges et lycées (précisez les disciplines)

- Formateurs IUFM

Nombre d'heures de formation:

Nombre de personnes en formation:

Nombre de formateurs intervenant dans ces formations :

Place dans les plans de formations

Les formations en HST sont assurées sur les moyens :

- Des formations disciplinaires des plans de formation?

- Des formations transversales?

Modalités des stages et formations en HST

- Etude de textes et documents

- Présentation, exposé, conférence

- Atelier de production de séquences d'enseignement

- Autre (précisez) :

Articulation formation en HST et formation disciplinaire

- Formation en HST comme discipline à part entière

- Formation en HST comme élément d'une culture scientifique personnelle

- Formation en HST au service de l'enseignement disciplinaire

- en liaison avec les aspects didactiques de la discipline

- en liaison avec les aspects épistémologiques de la discipline

- en liaison avec les aspects historiques de la discipline

\section{Annexe 2}


Tableau synoptique des réponses en ce qui concerne la formation initiale en Histoire des Sciences et des Techniques (HST)

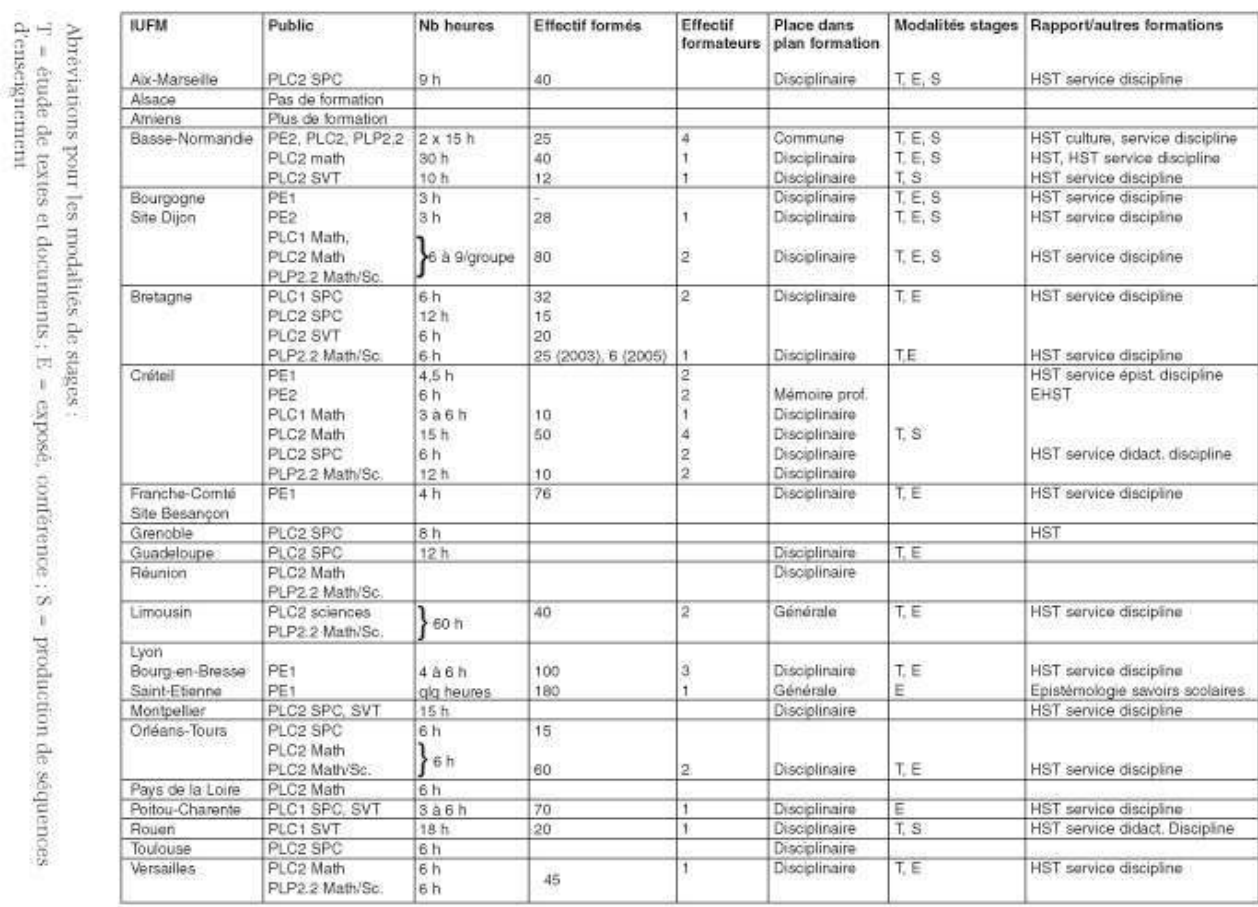

\section{Annexe 3}

Tableau synoptique des réponses en ce qui concerne la formation continue en Histoire des Sciences et des Techniques (HST)

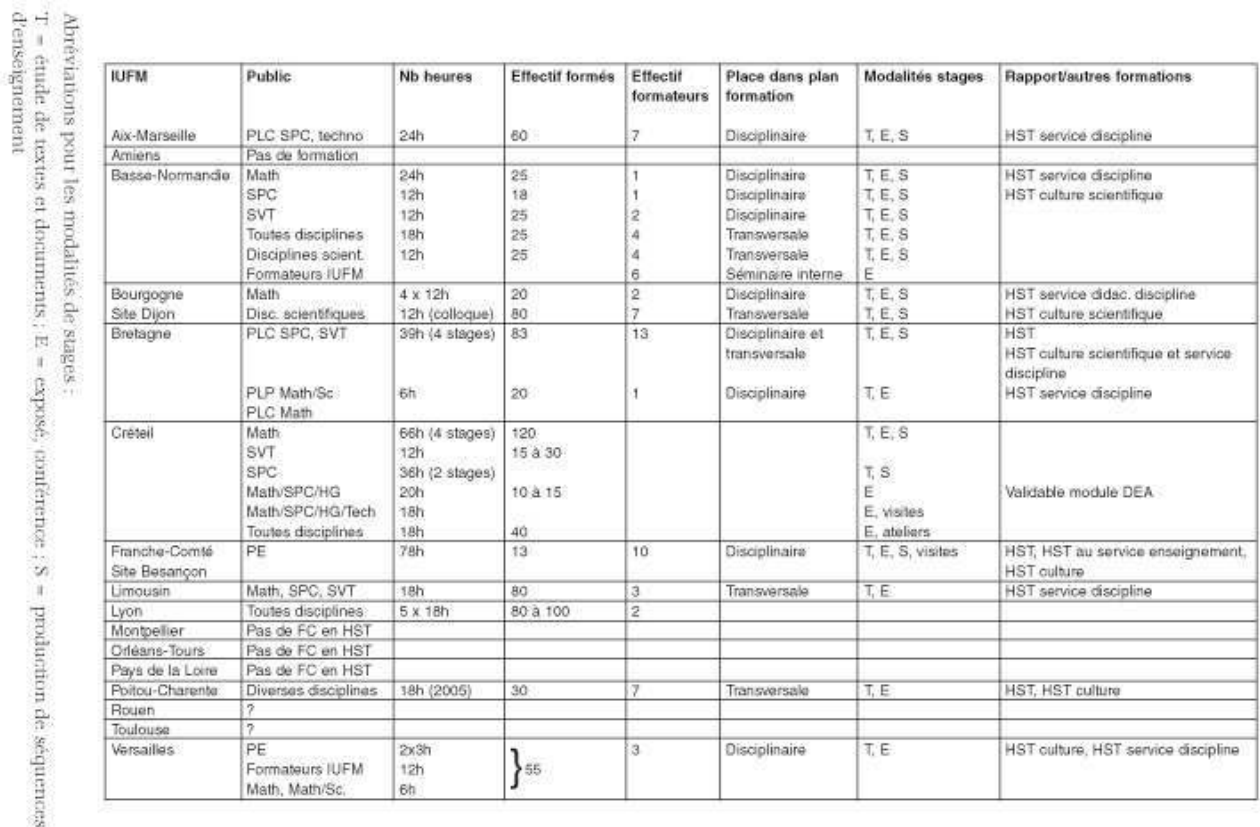




\section{NOTES}

1. Ces appellations officielles désignent : PE1, étudiants préparant le concours de professeurs d'écoles ; PE2, stagiaires professeurs d'écoles ; PLC1, étudiants préparant un concours pour l'obtention d'un CAPES ou certificat d'aptitude à l'enseignement secondaire, c'est à dire à l'enseignement en collèges et lycées ; PLC2, stagiaires professeurs de lycées et collèges ; PLP2.2, stagiaires professeurs de lycées professionnels.

2. $\mathrm{SVT}=$ Sciences de la Vie et de la Terre $; \mathrm{SPC}=$ Sciences physiques et chimiques. Il s'agit là des appellations officielles de ces enseignements dans le secondaire et dans la formation des maîtres. 3. Dans l'esprit fondateur des IUFM, les étudiants et les stagiaires devraient pouvoir suivre des formations en tant qu'étudiants de première année ou stagiaire de $2^{\mathrm{e}}$ année et pas seulement en tant que futur enseignant du Primaire ou du Secondaire, stagiaire de mathématiques ou de lettres classiques. Deux journées sur l'histoire des sciences et des techniques sont ainsi proposées à l'IUFM de Basse-Normandie à l'ensemble des stagiaires.

4. IREM = Institut de Recherche et d'Etudes en Mathématiques

\section{RÉSUMÉS}

L'article expose brièvement les résultats d'une enquête conduite en 2005 sur l'existence de formations en épistémologie et histoire des sciences et des techniques dans les IUFM. Les réponses montrent une forte diversité de situations entre IUFM, formations et disciplines avec un ancrage en PLC2 et en formation continue des enseignants du secondaire. Ces formations associent la plupart du temps des exposés et des études de textes.

This paper shortly exposes the results of an inquiry, realized in 2005, about trainings courses in epistemology and history of sciences and techniques in the IUFM. The answers show a big variety of situations between the IUFM, the trainings courses and the disciplines (mathematics, physics, chemistry, biology, geology and technology). When trainings exist, it's generaly for teachers of secundary level and combine conferences with texts studies.

\section{AUTEUR}

\section{PIERRE SAVATON}

Centre d'études et de recherche en sciences de l'éducation CERSE IUFM de Basse Normandie. pierre.savaton@wanadoo.fr 